\title{
Multilevel axicon for perfect optical vortex generation
}

\author{
Rebeca Tudor*, Mihai Kusko, Cristian Kusko, and Andrei Avram \\ National Institute for R\&D in Microtechnology IMT, Bucharest, 077190, Romania
}

\begin{abstract}
We present the fabrication of a beam shaper with 32 levels for the generation of nondiffractive optical fields representing quasi-Bessel beams of order zero. This optical element is designed for visible light $(\lambda=633 \mathrm{~nm})$ and fabricated using standard photolithography and a fine calibrated reactive ion etching process. A large number of levels approximates a continuous conical surface so that the optical quality of the element is very good. It is investigated the possibility of generating perfect optical vortices with this class of optical elements.
\end{abstract}

\section{Introduction}

Ideal Bessel beams are nondiffractive optical fields described by invariant intensity profiles along an infinite propagation distance. In experiments, one can generate only quasi-Bessel beams characterized by finite focal extensions and intensity profiles that vary along the propagation axis but only after a specific distance called focal length. Quasi-Bessel beams can be generated with conical lens, planar Pancharatnam-Berry phase elements, spatial light modulator, Fresnel lens-based elements [1-4]. Nondiffractive unique beams are widely exploited in fields like optical trapping [5], beam shaping [6-9] optical communications [10-11], microscopy [12].

\section{Fabrication process}

In this work we designed, fabricated and characterized a diffractive axicon as a phase control element in order to obtain nondiffractive optical fields for visible light. The design was considered an approximation of continuous conical surface in order to generate a Fresnel based element presenting a discrete surface defined by 32 levels. This element was fabricated on a silicon wafer using standard photolithography and a fine calibrated reactive ion etching process. The multilevel diffractive optical element generates quasi-Bessel beams of order zero.

\subsection{Structural characterization}

We investigated the optical quality of the fabricated axicon with the optical microscope. In Fig. 1 is represented a small area of an axicon of order zero with 32 levels. Red colour represents the maximum level and the green colour indicate the minimum level. The step height between the maximum and minimum level introduces a phase shift in the wavefront of an incident Gaussian beam in order to generate nondiffractive beams.

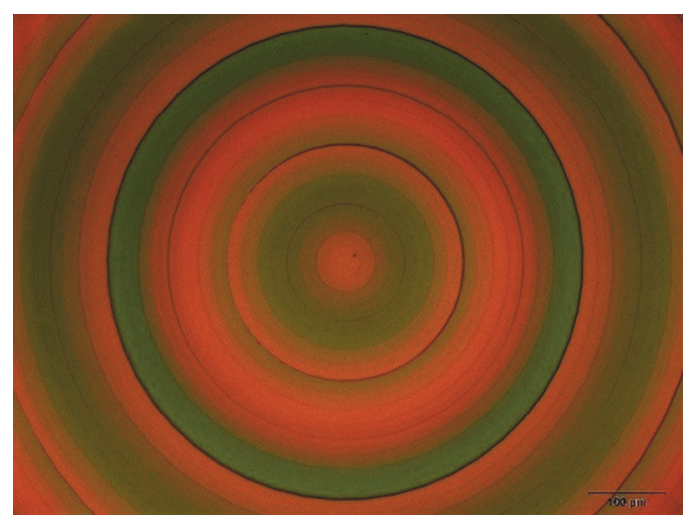

Fig. 1. Optical image of an axicon of order zero with 32 levels

\subsection{Functional characterization}

We beam shaped a Gaussian beam with the wavelength $\lambda=633 \mathrm{~nm}$ using the fabricated axicon and generated a quasi-Bessel beam with order zero which is illustrated in the Fig. 2. One can observe the intensity distribution characterized by a central lobe surrounded by concentric rings.

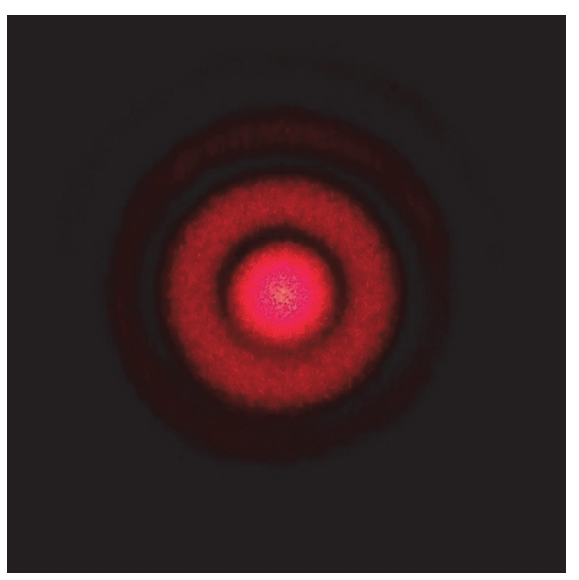

Fig. 2. Intensity distribution for a Bessel beam of order zero

\footnotetext{
* Corresponding author: rebeca.tudor@imt.ro
} 


\subsection{Conclusions}

We designed, fabricated and characterized a multilevel beam shaper for visible light $(\lambda=633 \mathrm{~nm})$ in order to generate a quasi-Bessel beam of order zero. We obtained a diffractive axicon with a very good approximation of a continuous surface by increasing the number of levels. This was possible due to multiple photolitographical and reactive ion etching processes. The structural and functional characterization confirmed the optical quality of the fabricated axicon with 32 levels. This optical element has potential industrial application in the generation of perfect optical vortices [10], beam shaping, optical communications and optical imaging.

\section{References}

1. I. Golub, Opt Lett. 31(12), (2006)

2. Y. Liu, Y. Ke, J. Zhou, Y. Liu, H. Luo, S. Wen, D. Fan, Sci. Rep 7 (2017)

3. R. Li, X. Yu, T. Peng, Y. Yang, B. Yao, C. Zhang, T. Ye, J. Opt. 20085603 (2018)

4. D.J. Fischer, C.J. Harkrider, D.T. Moore, Appl. Opt., AO. 39 2687-2694 (2000)

5. V. Garces-Chavez, D. McGloin, H. Melville, W. Sibbett, K. Dholakia Nature 419 (2002)

6. L. Shi, X. Dong, Q. Deng, Y. Lu, Y. Ye, C. Du, Opt. Eng 50 (2011)

7. D. Deng, Y. Li, Y. Han, X. Su, J. Ye, J. Gao, Q. Sun, S. Qu, OE. 24 (2016)

8. S. Khonina, S. Degtyarev, D. Savelyev, A. Ustinov, OE. 25 (2017)

9. S. Supp, J. Jahns, JEOS-RP. 14 (2018)

10. A. Burvall, P. Martinsson, A.T. Friberg, OE. 12 (2004)

11. S. Chen, S. Li, Y. Zhao, J. Liu, L. Zhu, A. Wang, J. Du, L. Shen, J. Wang, OL. 41 (2016)

12. F. O. Fahrbach, A. Rohrbach Opt. Exp 18 (2010)

\section{Acknowlegdement}

The authors would like to acknowledge the financial support from IMT-Bucharest Core Programme "MICRONANO-SIS" PN 4N/2018 and "MICRO-NANO-SISPLUS" PN 14N/2018 supported by MCI. 